\title{
Observation of a Direct Correlation of the Crystallite Morphology and the Optical Properties in Indium Tin Oxide Thin Films
}

\author{
Asher C. Leff ${ }^{*}$, Wendy L. Sarney ${ }^{1}$, Jimmy Ni $^{1}$ and Weimin Zhou ${ }^{1}$ \\ 1. US Army Combat Capabilities Development Command, Army Research Laboratory, Sensors and \\ Electron Devices Directorate, Adelphi, MD, USA. \\ * Corresponding author: asher.c.leff.ctr@mail.mil
}

Previous studies demonstrated indium tin oxide (ITO) thin films with epsilon-near-zero (ENZ) for wavelengths in the infrared (IR) regimes [1]. These films have potential for use in waveguide supercoupling, nonlinear optics, and ultrafast switching devices [2-4]. So far, they have been limited to film thicknesses of a few hundred nanometers or less. To produce devices that function at the standard wavelengths used for telecommunications $(1310 \mathrm{~nm}$ and $1550 \mathrm{~nm})$, ENZ films of at least the same thickness as those wavelengths must be produced. Working towards that goal, we grew $2 \mu \mathrm{m}$ films of ITO via sputter deposition with a nominal ratio of $90 / 10 \mathrm{In}_{2} \mathrm{O}_{3} / \mathrm{SnO}_{2}$ by weight and evaluated the effects of various annealing treatments on the microstructure and optical properties.

The ITO films were evaluated using ellipsometry, x-ray diffraction (XRD), and transmission electron microscopy (TEM). The as-deposited ITO films were predominantly amorphous as observed in TEM, however some crystallinity was observed via XRD. Since even short time periods of localized sample heating by the electron beam crystallizes the sample, it was not possible to determine the location and extent of the crystallinity in the as-grown film.

After annealing for 10 minutes at $350{ }^{\circ} \mathrm{C}$, the film was fully crystalline with a cubic Bixbyite structure (space group 206: Iwith a lattice parameter of $1.013 \mathrm{~nm}$. Ellipsometry shows that there is a transition in the permittivity halfway through the thickness of the film with a positive $\varepsilon$ in the top half and a negative $\varepsilon$ in the bottom half (Fig. 1a). TEM imaging revealed that there is a distinct crystal morphology difference between the top and bottom half of the film corresponding exactly to the change in $\varepsilon$. As shown in the STEM-HAADF image in Fig. 1a, the bottom half of the film has an equiaxed grain structure with an average grain diameter of $160 \mathrm{~nm}$, while the top half of the film has a columnar grain structure with significant porosity evident between the grains. However, both XRD and selected area electron diffraction show that there is no change in the crystal structure or lattice parameter (Fig. 1b-d). Furthermore, we etched away the columnar portion of the annealed ITO film and found no significant change in the XRD. These findings indicate that controlling the crystal morphology is a critical parameter for tuning the permittivity of ITO in order to produce micron-scale ENZ films.

\section{References:}

[1] JW Cleary et al., Optical Materials Express 8 (2018), p. 1231.

[2] I Liberal and N Engheta, Proceedings of the National Academy of Sciences, 114 (2017), p. 822.

[3] AM Mahmoud and N Engheta, Nature Communications 5 (2014), p. 5638

[4] N Kinsey et al., Optica 2 (2015), p. 616. 

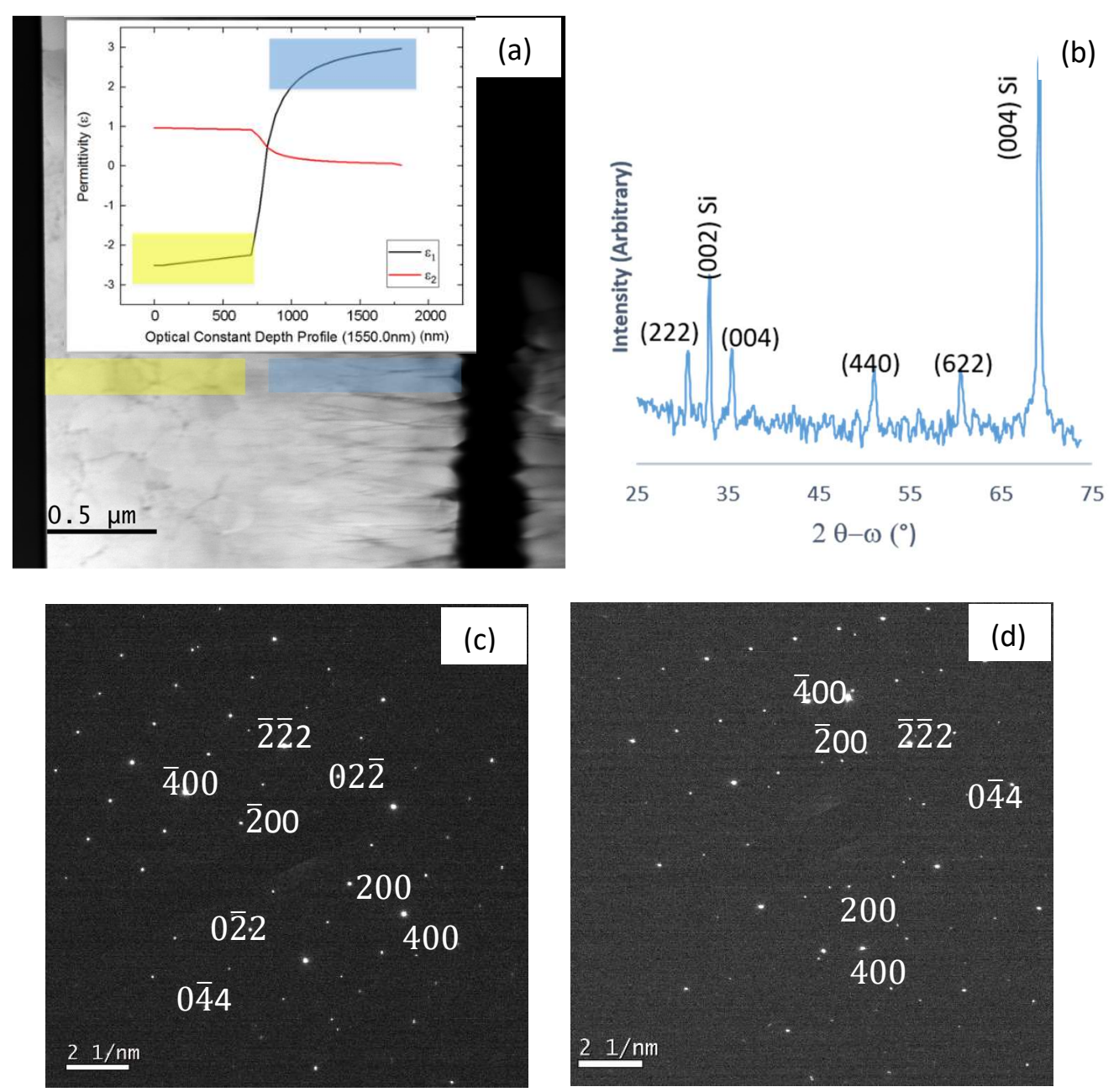

Figure 1. a) STEM-HAADF micrograph showing two distinct grain morphologies across the crosssection of the annealed ITO film with a plot of the permittivity as a function of depth overlaid. b) XRD data from the annealed ITO film with peaks corresponding to the Bixbyite structure. c \& d) [110] selected area diffraction patterns from an equiaxed and a columnar grain, respectively, indicating the same crystal structure and lattice parameter for both regions. Stray spots are visible due to diffraction from neighboring grains and/or along the beam direction. 